Electronic Journal of Statistics

Vol. 4 (2010) 585-605

ISSN: $1935-7524$

DOI: $10.1214 / 09-E J S 548$

\title{
Discrete temporal models of social networks
}

\author{
Steve Hanneke \\ Department of Statistics, Carnegie Mellon University \\ Pittsburgh, PA 15213 USA \\ e-mail: shanneke@stat.cmu.edu \\ Wenjie Fu \\ School of Computer Science, Carnegie Mellon University \\ Pittsburgh, PA 15213 USA \\ e-mail: wenjief@cs.cmu.edu \\ Eric P. Xing* \\ School of Computer Science, Carnegie Mellon University \\ Pittsburgh, PA 15213 USA \\ e-mail: epxing@cs.cmu.edu
}

\begin{abstract}
We propose a family of statistical models for social network evolution over time, which represents an extension of Exponential Random Graph Models (ERGMs). Many of the methods for ERGMs are readily adapted for these models, including maximum likelihood estimation algorithms. We discuss models of this type and their properties, and give examples, as well as a demonstration of their use for hypothesis testing and classification. We believe our temporal ERG models represent a useful new framework for modeling time-evolving social networks, and rewiring networks from other domains such as gene regulation circuitry, and communication networks.
\end{abstract}

Received November 2009

\section{Introduction}

The field of social network analysis is concerned with populations of actors, interconnected by a set of relations (e.g., friendship, communication, etc.). These relationships can be concisely described by directed graphs, with one vertex for each actor and an edge for each relation between a pair of actors. This network representation of a population can provide insight into organizational structures, social behavior patterns, emergence of global structure from local dynamics, and a variety of other social phenomena.

There has been increasing demand for flexible statistical models of social networks, for the purposes of scientific exploration and as a basis for practical analysis and data mining tools. The subject of modeling a static social network has been investigated in some depth. For time-invariant networks, represented

\footnotetext{
${ }^{*}$ To whom correspondence should be addressed.
} 
as a single directed or undirected graph, a number of flexible statistical models have been proposed, including the classic Exponential Random Graph Models (ERGM) and extensions (Frank and Strauss, 1986; Wasserman and Robins, 2005; Snijders, 2002; Robins and Pattison, 2005), which are descriptive in nature, latent space models that aim towards clustering and community discovery (Handcock et al., 2007), and mixed-membership block models for role discovery (Airoldi et al., 2008). Of particular relevance to this paper is the ERGM, which is particularly flexible in that it can be customized to capture a wide range of signature connectivity patterns in the network via user-specified functions representing their sufficient statistics. Specifically, if $N$ is some representation of a social network, and $\mathcal{N}$ is the set of all possible networks in this representation, then the probability distribution function for any ERGM can be written in the following general form.

$$
\mathcal{P}(N)=\frac{1}{Z(\boldsymbol{\theta})} \exp \left\{\boldsymbol{\theta}^{\prime} \mathbf{u}(N)\right\}
$$

Here, $\boldsymbol{\theta} \in \mathbb{R}^{k}$, and $\mathbf{u}: \mathcal{N} \rightarrow \mathbb{R}^{k} . Z(\boldsymbol{\theta})$ is a normalization constant, which is typically intractable to compute. The $\mathbf{u}$ function represents the sufficient statistics for the model, and, in a graphical modeling interpretation, can be regarded as a vector of clique potentials. The representation for $N$ can vary widely, possibly including multiple relation types, valued or binary relations, symmetric or asymmetric relations, and actor and relation attributes. The most widely studied models of this form are for single-relation social networks, in which case $N$ is generally taken to be the weight matrix $A$ for the network (sometimes referred to as a sociomatrix), where $A_{i j}$ is the strength of directed relation between the $i^{\text {th }}$ actor and $j^{\text {th }}$ actor.

Often one is interested in modeling the evolution of a network over multiple sequential observations. For example, one may wish to model the evolution of coauthorship networks in a specific community from year to year, trends in the evolution of the World Wide Web, or a process by which simple local relationship dynamics give rise to global structure. In such dynamic settings, where a timeseries of observations of the network structure is available, several formalisms have been proposed to model the dynamics of topological changes of such networks over time. For example, Snijders (2006) has proposed a continuous-time model of network dynamics, where each observed event represents a single actor altering his or her outgoing links to optimize an objective function based on local neighborhood statistics. Robins and Pattison (2001) have indepedently studied a family of models of network dynamics over discrete time steps, quite similar to those presented below; in some sense, the present work can be viewed as a further exploration of these models, their properties and uses. However, this exploration goes beyond the (Robins and Pattison, 2001) work, in that we explore the statistical properties of these models, such as their (non)degeneracy tendencies, and the quality of fit that these models achieve when applied to real network time series data; such properties have not previously been systematically investigated for these types of models, though related work has recently 
been done on static ERGMs (Handcock, 2003). We also explore algorithmic issues in calculating the MLE estimators and performing hypothesis tests with these models. Furthermore, we feel that the added flexibility in the parametrization of these models below makes them somewhat easier to specify and work with, compared to the description in (Robins and Pattison, 2001), which although quite elegant, requires the sufficient statistics to be nondecreasing in the relation indicator variables for the network.

In the following sections, we propose a model family we would like to refer to as temporal ERGM, or TERGM, that is capable of modeling network evolution, while maintaining the flexibility of a fully general ERGM. Furthermore, these models build upon a generic ERGM formalism, so that existing methods developed for ERGMs over the past two decades are readily adapted to apply to the temporal models as well. We prove that a very general subclass of the TERGM is nondegenerate and explain how to calculate their maximum likelihood estimates from network data. Furthermore we show that these models can indeed be fitted to capture signature dynamic properties of real world evolving networks, and can be applied in hypothesis testing, nodal classification, and other applications.

\section{Discrete temporal models}

We begin by describing the basic form of the type of model we study. Specifically, one way to simplify a statistical model for evolving social networks is to make a Markov assumption on the network from one time step to the next. If $A^{t}$ is the weight matrix representation of a single-relation social network at time $t$, then we might make the assumption that $A^{t}$ is independent of $A^{1}, \ldots, A^{t-2}$ given $A^{t-1}$. Put another way, a sequence of network observations $A^{1}, \ldots, A^{t}$ has the property that

$$
\mathcal{P}\left(A^{2}, A^{3}, \ldots, A^{t} \mid A^{1}\right)=\mathcal{P}\left(A^{t} \mid A^{t-1}\right) \mathcal{P}\left(A^{t-1} \mid A^{t-2}\right) \cdots \mathcal{P}\left(A^{2} \mid A^{1}\right) .
$$

With this assumption in mind, we can now set about deciding what the form of the conditional PDF $\mathcal{P}\left(A^{t} \mid A^{t-1}\right)$ should be. Given our Markov assumption, one natural way to generalize ERGMs for evolving networks is to assume $A^{t} \mid A^{t-1}$ admits an ERGM representation. That is, we can specify a function $\Psi: \mathbb{R}_{n \times n} \times$ $\mathbb{R}_{n \times n} \rightarrow \mathbb{R}^{k}$, which can be understood as a temporal potential over cliques across two time-adjancent networks, and parameter vector $\boldsymbol{\theta} \in \mathbb{R}^{k}$, such that the conditional PDF has the following form.

$$
\mathcal{P}\left(A^{t} \mid A^{t-1}, \boldsymbol{\theta}\right)=\frac{1}{Z\left(\boldsymbol{\theta}, A^{t-1}\right)} \exp \left\{\boldsymbol{\theta}^{\prime} \boldsymbol{\Psi}\left(A^{t}, A^{t-1}\right)\right\} .
$$

We refer to such a model as a TERGM, for Temporal Exponential Random Graph Model. Note that specifying the joint distribution requires one to specify a distribution over the first network: $A^{1}$. This can generally be accomplished fairly naturally using an ERGM. For simplicity of presentation, we avoid these details 
in subsequent sections by assuming the distribution over this initial network is functionally independent of the parameter $\boldsymbol{\theta}$.

In particular, we will be especially interested in the special case of these models in which

$$
\boldsymbol{\Psi}\left(A^{t}, A^{t-1}\right)=\sum_{i j} \boldsymbol{\Psi}_{i j}\left(A_{i j}^{t}, A^{t-1}\right) .
$$

This form of the temporal potential function represents situations where the conditional distribution of $A^{t}$ given $A^{t-1}$ factors over the entries $A_{i j}^{t}$ of $A^{t}$. As we will see, such models possess a number of desirable properties.

\subsection{An example}

To illustrate the expressiveness of this framework, we present the following simple example model. For simplicity, assume the weight matrix of the network is binary (i.e., an adjacency matrix). Define the following statistics, which represent density, stability, reciprocity, and transitivity, respectively:

$$
\begin{aligned}
& \Psi_{D}\left(A^{t}, A^{t-1}\right)=\frac{1}{(n-1)} \sum_{i j} A_{i j}^{t} \\
& \Psi_{S}\left(A^{t}, A^{t-1}\right)=\frac{1}{(n-1)} \sum_{i j}\left[A_{i j}^{t} A_{i j}^{t-1}+\left(1-A_{i j}^{t}\right)\left(1-A_{i j}^{t-1}\right)\right] \\
& \Psi_{R}\left(A^{t}, A^{t-1}\right)=n\left[\sum_{i j} A_{j i}^{t} A_{i j}^{t-1}\right] /\left[\sum_{i j} A_{i j}^{t-1}\right] \\
& \Psi_{T}\left(A^{t}, A^{t-1}\right)=n\left[\sum_{i j k} A_{i k}^{t} A_{i j}^{t-1} A_{j k}^{t-1}\right] /\left[\sum_{i j k} A_{i j}^{t-1} A_{j k}^{t-1}\right] .
\end{aligned}
$$

The statistics are each scaled to a constant range (in this case $[0, n]$ ) to enhance interpretability of the model parameters (take $0 / 0=0$ for our purposes). The conditional probability mass function (1) is governed by four parameters: $\theta_{D}$ relates to the density, or the number of ties in the network as a whole; $\theta_{S}$ relates to the stability, or the tendency of a link that does (or does not) exist at time $t-1$ to continue existing (or not existing) at time $t ; \theta_{R}$ relates to the reciprocity, or the tendency of a link from $i$ to $j$ to result in a link from $j$ to $i$ at the next time step; and $\theta_{T}$ relates to the transitivity, or the tendency of a tie from $i$ to $j$ and from $j$ to $k$ to result in a tie from $i$ to $k$ at the next time step. The transition probability for this temporal network model can then be expressed as

$$
\mathcal{P}\left(A^{t} \mid A^{t-1}, \boldsymbol{\theta}\right)=\frac{1}{Z\left(\boldsymbol{\theta}, A^{t-1}\right)} \exp \left\{\sum_{j \in\{D, S, R, T\}} \theta_{j} \Psi_{j}\left(A^{t}, A^{t-1}\right)\right\} .
$$




\subsection{More general models}

For simplicity, we will only discuss the simple models described above. However, one can clearly extend this framework to allow multiple relations in the network, actor attributes, relation attributes, longer-range Markov dependencies, or a host of other possibilities. In fact, many of the results below can easily be generalized to deal with these types of extensions.

\section{Estimation}

The estimation task for models of the form (1) is to use the sequence of observed networks, $N^{1}, N^{2}, \ldots, N^{T}$, to find an estimator $\hat{\boldsymbol{\theta}}$ that is close to the actual parameter values $\boldsymbol{\theta}$ in some sensible metric. As with ERGMs, in general the normalizing constant $Z$ could be computationally intractable, often making explicit solutions of maximum likelihood estimation difficult. However, general techniques for MCMC sampling to enable approximate maximum likelihood estimation for ERGMs have been studied in some depth and have proven successful for a variety of models (Snijders, 2002). By a slight modification of these algorithms, we can apply the same general techniques as follows.

Let

$$
\begin{gathered}
\mathcal{L}\left(\boldsymbol{\theta} ; N^{1}, N^{2}, \ldots, N^{T}\right)=\log \mathcal{P}\left(N^{2}, N^{3}, \ldots, N^{T} \mid N^{1}, \boldsymbol{\theta}\right), \\
\mathbf{M}(t, \boldsymbol{\theta})=\mathbb{E}_{\boldsymbol{\theta}}\left[\Psi\left(\underline{\mathbf{N}}^{t}, N^{t-1}\right) \mid N^{t-1}\right], \\
\mathbf{C}(t, \boldsymbol{\theta})=\mathbb{E}_{\boldsymbol{\theta}}\left[\Psi\left(\underline{\mathbf{N}}^{t}, N^{t-1}\right) \Psi\left(\underline{\mathbf{N}}^{t}, N^{t-1}\right)^{\prime} \mid N^{t-1}\right] .
\end{gathered}
$$

where expectations are taken over the random variable $\underline{\mathbf{N}}^{t}$, the network at time $t$. Note that

$$
\nabla \mathcal{L}\left(\boldsymbol{\theta} ; N^{1}, \ldots, N^{T}\right)=\sum_{t=2}^{T}\left(\Psi\left(N^{t}, N^{t-1}\right)-M(t, \boldsymbol{\theta})\right)
$$

and

$$
\nabla^{2} \mathcal{L}\left(\boldsymbol{\theta} ; N^{1}, \ldots, N^{T}\right)=\sum_{t=2}^{T}\left(M(t, \boldsymbol{\theta}) M(t, \boldsymbol{\theta})^{\prime}-C(t, \boldsymbol{\theta})\right) .
$$

The expectations can be approximated by Gibbs sampling from the conditional distributions (Snijders, 2002), so that we can perform an unconstrained optimization procedure akin to Newton's method: approximate the expectations, update parameter values in the direction that increases (3), repeat until convergence. A related algorithm is described by (Geyer and Thompson, 1992) for general exponential families, and variations are given by (Snijders, 2002) that are tailored for ERG models. The following is a simple version of such an estimation algorithm. 


$$
\begin{aligned}
& \text { 1. } \text { Randomly initialize } \boldsymbol{\theta}^{(1)} \\
& \text { 2. } \text { For } i=1 \text { up until convergence } \\
& \text { 3. } \quad \text { For } t=2,3, \ldots, T \\
& \text { 4. } \quad \text { Sample } \hat{N}_{(i)}^{t, 1}, \ldots, \hat{N}_{(i)}^{t, B} \sim \mathcal{P}\left(\underline{\mathbf{N}}^{t} \mid N^{t-1}, \boldsymbol{\theta}^{(i)}\right) \\
& \text { 5. } \quad \hat{\mu}_{(i)}^{t}=\frac{1}{B} \sum_{b=1}^{B} \Psi\left(\hat{N}_{(i)}^{t, b}, N^{t-1}\right) \\
& \text { 6. } \quad \hat{C}_{(i)}^{t}=\frac{1}{B} \sum_{b=1}^{B} \mathbf{\Psi}\left(\hat{N}_{(i)}^{t, b}, N^{t-1}\right) \Psi\left(\hat{N}_{(i)}^{t, b}, N^{t-1}\right)^{\prime} \\
& \text { 7. } \quad \hat{H}_{(i)}=\sum_{t=2}^{T}\left[\hat{\mu}_{(i)}^{t} \hat{\mu}_{(i)}^{t \prime}-\hat{C}_{(i)}^{t}\right] \\
& \text { 8. } \boldsymbol{\theta}^{(i+1)} \leftarrow \boldsymbol{\theta}^{(i)}-\hat{H}_{(i)}^{-1} \sum_{t=2}^{T}\left[\Psi\left(N^{t}, N^{t-1}\right)-\hat{\mu}_{(i)}^{t}\right]
\end{aligned}
$$

The choice of $B$ can affect the convergence of this algorithm. Generally, larger $B$ values will give more accurate updates, and thus fewer iterations needed until convergence. However, in the early stages of the algorithm, precise updates might not be necessary if the likelihood function is sufficiently smooth, so that a $B$ that grows larger only when more precision is needed may be appropriate. If computational resources are limited, it is possible (though less certain) that the algorithm might still converge even for small $B$ values (see (Carreira-Perpignán and Hinton, 2005) for an alternative approach to samplingbased MLE, which seems to remain effective for small $B$ values).

\subsection{Product transition probabilities}

Although the general case (1) may often require a sampling-based estimation procedure such as that given above, it turns out that the special case of (2) does not. In this case, as long as the $\boldsymbol{\Psi}_{i j}$ functions are computationally tractable, we can tractably perform exact updates in Newton's method, rather than approximating them with sampling.

\subsection{Evalutation of parameter recovery}

To examine the convergence rate empirically, we display in Figure 1 the convergence of this algorithm on data generated from the example model given in Section 2.1. The simulated data is generated by sampling from the example model with randomly generated $\boldsymbol{\theta}$, and the loss is plotted in terms of Euclidean distance of the estimator from the true parameters. To generate the initial $N^{1}$ network, we sample from the $\operatorname{pmf} \frac{1}{Z(\boldsymbol{\theta})} \exp \left\{\boldsymbol{\theta}^{\prime} \boldsymbol{\Psi}\left(N^{1}, N^{1}\right)\right\}$. The number of actors $n$ is 100 . The parameters are initialized uniformly in the range $[0,10)$, except for $\theta_{D}$, which is initialized to $-5 \theta_{S}-5 \theta_{R}-5 \theta_{T}$. This tends to generate networks with reasonable densities. The results in Figure 1 represent averages over 10 random initial configurations of the parameters and data. In the estimation algorithm used, $B=100$, but increases to 1000 when the Euclidean distance between parameter estimates from the previous two iterations is less than 1 . Convergence is defined as the Euclidean distance between $\boldsymbol{\theta}^{(i+1)}$ and $\boldsymbol{\theta}^{(i)}$ being within 0.1. Since this particular model is simple enough for exact calculation 


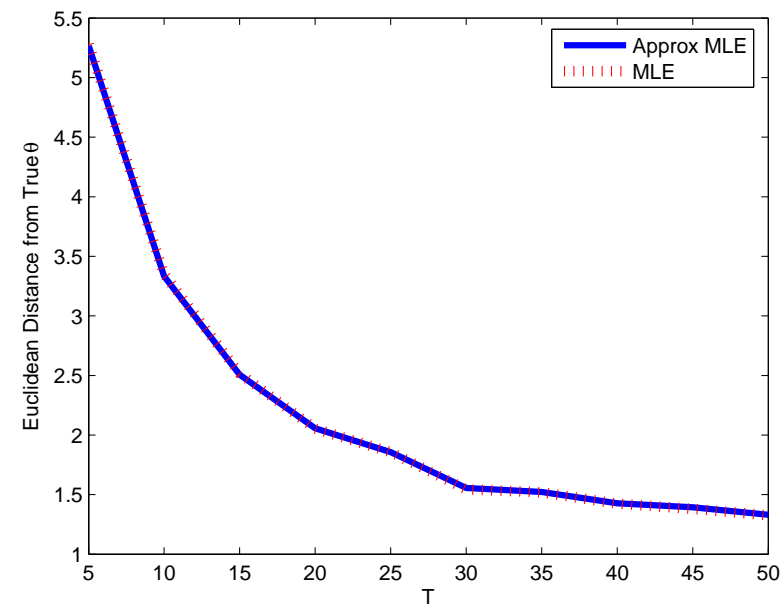

FIG 1. Convergence of estimation algorithm on simulated data, measured in Euclidean distance of the estimated values from the true parameter values. The approximate MLE from the sampling-based algorithm is almost identical to the MLE obtained by direct optimization.

of the likelihood and derivatives thereof (see above), we also compare against Newton's method with exact updates (rather than sampling-based). We can use this to determine how much of the loss is due to the approximations being performed, and how much of it is intrinsic to the estimation problem. The parameters returned by the sampling-based approximation are usually almost identical to the MLE obtained by Newton's method, and this behavior manifests itself in Figure 1 by the losses being visually indistinguishable.

\section{4. (Non)degeneracy of temporal ERGMs}

There has been much concern expressed in the literature over certain degeneracy issues that arise when working with Exponential Random Graph Models. Specifically, Handcock (Handcock, 2003) has recently been exploring the fact that, for many ERGMs, most of the parameter space is populated by distributions that place almost all of the probability mass on a small number of networks (typically the complete or empty graphs). This leads to several negative effects. For instance, since we do not expect the true generating distribution to be degenerate, the prevalence of these degenerate distributions intuitively reflects a mismatch between the model and the type of process we wish to capture with it. Additionally, it is often the case that degenerate distributions can be found very close to any nondegenerate distribution, so that slight variations in the parameters cause the distribution to become degenerate. This also leads to another problem, namely inference degeneracy. Even if the generating distribution is modeled by some parameter values with a nondegenerate distribution, the degeneracy of nearby distributions may prevent commonly used maximum likelihood estimation techniques from converging to it within a reasonable sample 
size; specifically, the aforementioned MCMC techniques may require an impractically large number of samples, or may even fail to work at all (Handcock, 2003).

One natural question to ask is whether such issues also affect these temporal extensions. In the simple case where the transition distribution factors over the edges, as in (2), it turns out these models avoid such problems entirely. The intuitive reason for this is that, since the edges of $A^{t}$ are conditionally independent given $A^{t-1}$, as long as the individual conditional distributions for the $A_{i j}^{t}$ given $A^{t-1}$ are not too extreme, the conditional entropy of $A^{t}$ given $A^{t-1}$ should be large, and thus the entropy of $A^{t}$ itself must be large. Of course, this argument only works if the dependence of $A^{t}$ on $A^{t-1}$ is not too strong, and the strength of this dependence can be controlled by the magnitudes of the parameters. The formal argument is given below.

\subsection{Nondegeneracy of the example model}

To keep the initial explanation of this phenomenon as simple as possible, we begin this discussion by looking at the special case of the example model from Section 2.1.

For any given entry $A_{i j}^{t}$, the networks $A^{t-1}$ that minimize and maximize the probability that $A_{i j}^{t}=1$ are the empty graph and complete graph; which one maximizes and which one minimizes it depends on the parameter values. If $A^{t-1}$ is the empty graph, then

$$
\mathcal{P}\left(A_{i j}^{t}=1 \mid A^{t-1}\right)=\frac{\exp \left\{\theta_{D} /(n-1)\right\}}{\exp \left\{\theta_{D} /(n-1)\right\}+\exp \left\{\theta_{S} /(n-1)\right\}} .
$$

Under $A^{t-1}$ as the complete graph, it is

$$
\mathcal{P}\left(A_{i j}^{t}=1 \mid A^{t-1}\right)=\frac{\exp \left\{\left(\theta_{D}+\theta_{S}+\theta_{T}+\theta_{R}\right) /(n-1)\right\}}{\exp \left\{\left(\theta_{D}+\theta_{S}+\theta_{T}+\theta_{R}\right) /(n-1)\right\}+1} .
$$

So the entropy is lower bounded as follows:

$$
\begin{aligned}
H\left(A^{t}\right) & \geq \min _{A} H\left(A^{t} \mid A^{t-1}=A\right) \\
& =\min _{A} \sum_{i j} H\left(A_{i j}^{t} \mid A^{t-1}=A\right) \geq \sum_{i j} \min _{A} H\left(A_{i j}^{t} \mid A^{t-1}=A\right) .
\end{aligned}
$$

We can lower bound $\min _{A} H\left(A_{i j}^{t} \mid A^{t-1}=A\right)$ by the quantity

$$
p \ln \frac{1}{p}+(1-p) \ln \frac{1}{1-p}
$$

where

$$
\begin{aligned}
p & =\frac{\exp \left\{\left(\left|\theta_{D}\right|+\left|\theta_{S}\right|+\left|\theta_{R}\right|+\left|\theta_{T}\right|\right) /(n-1)\right\}}{\exp \left\{\left(\left|\theta_{D}\right|+\left|\theta_{S}\right|+\left|\theta_{R}\right|+\left|\theta_{T}\right|\right) /(n-1)\right\}+\exp \left\{-\left(\left|\theta_{D}\right|+\left|\theta_{S}\right|+\left|\theta_{R}\right|+\left|\theta_{T}\right|\right) /(n-1)\right\}} \\
& =\frac{1}{\exp \left\{-2\left(\left|\theta_{D}\right|+\left|\theta_{S}\right|+\left|\theta_{R}\right|+\left|\theta_{T}\right|\right) /(n-1)\right\}+1} .
\end{aligned}
$$


( $p$ is an upper bound on $\mathcal{P}\left(A_{i j}^{t}=1 \mid A^{t-1}\right)$, and $1-p$ is a lower bound on it). So the entropy lower bound is at most $n(n-1)(p \ln (1 / p)+(1-p) \ln (1 /(1-p)))$, and thus as long as $\left|\theta_{D}\right|+\left|\theta_{S}\right|+\left|\theta_{R}\right|+\left|\theta_{T}\right|$ is not too large, the entropy is guaranteed to be reasonably large.

Other than the entropy, we can get a somewhat more intuitive grasp of this type of nondegeneracy result by bounding the expected number of nonzero entries in $A^{t}$. In particular, a consequence of the above reasoning is that the expected number of nonzero entries in $A^{t}$ is at most

$$
n(n-1) \frac{1}{\exp \left\{-2\left(\left|\theta_{D}\right|+\left|\theta_{S}\right|+\left|\theta_{R}\right|+\left|\theta_{T}\right|\right) /(n-1)\right\}+1},
$$

and is at least

$$
n(n-1) \frac{1}{\exp \left\{2\left(\left|\theta_{D}\right|+\left|\theta_{S}\right|+\left|\theta_{R}\right|+\left|\theta_{T}\right|\right) /(n-1)\right\}+1} .
$$

So again, as long as $\left|\theta_{D}\right|+\left|\theta_{S}\right|+\left|\theta_{R}\right|+\left|\theta_{T}\right|$ is not too large, we are guaranteed a reasonable expected number of nonzero entries in $A^{t}$.

To give an example of the types of entropy values one gets from a model of this type, in Figure 2 we plot the exact entropy values for the example model as a function of $\theta_{D}$ and $\theta_{S}$ (with $\theta_{R}=\theta_{T}=0$ to make a two-dimensional plot possible), and as a function of $\theta_{D}$ and $\theta_{T}$ (with $\theta_{R}=\theta_{S}=0$ ); other options, such as fixing the unused parameters to nonzero values, yield similar plots. Specifically, the plotted values are the entropy of $A^{2}$, where each $A^{i}$ is a $n \times n$ matrix (where $n=7$ in the left plot, and $n=6$ in the right plot), and $A^{1}$ is sampled from the basic Bernoulli graph model, in which each entry $A_{i j}^{1}$ is an independent Bernoulli random variable with probability of being 1 equal to 0.25 .

It is worth briefly mentioning how these plots are generated. Since it is not computationally tractable to enumerate all graphs for each parameter setting,
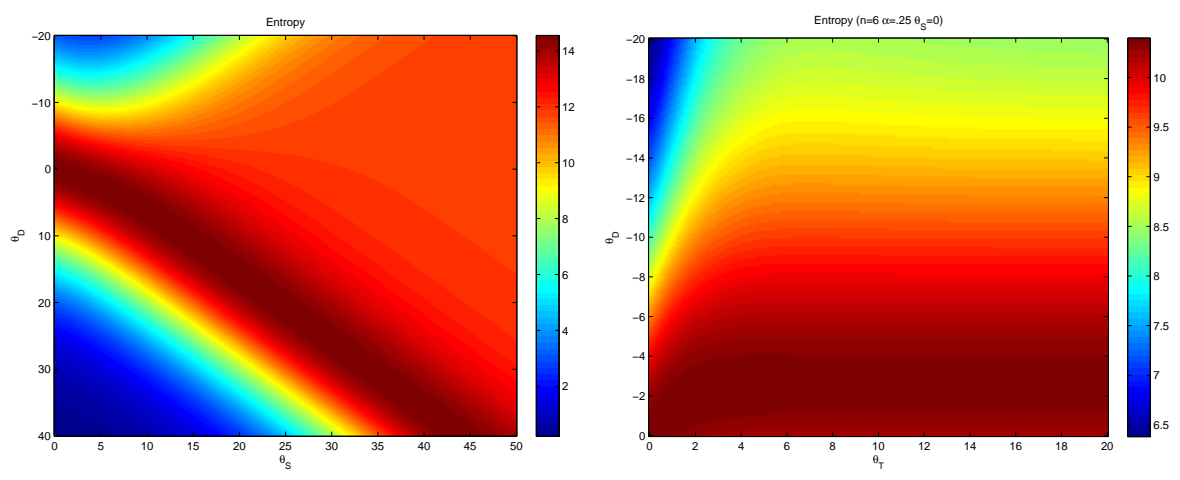

FIG 2. Entropy plots for the example model. In both plots, small magnitudes of the parameters give distributions with high entropy, as predicted. 
we instead calculate it for equivalence classes of graphs which can be analytically shown to have identical probability values, and weight each class according to its size in the entropy calculation. For the first plot, since the conditional probability of $A^{2}$ given $A^{1}$ is only a function of how many edges are present in $A^{2}$ and how many $i j$ values have $A_{i j}^{2}=A_{i j}^{1}$, and since the edges of $A^{1}$ are exchangeable, we can write the marginal distribution of $A^{2}$ purely in terms of the number of edges. Thus we need only calculate $n(n-1)$ probability values, and the entropy is a weighted sum, where the weights are combinatorial quantities reflecting the number of graphs with that many edges. For the second plot, the situation is more complex but the idea is similar. In this case, we define the equivalence classes based purely on graph isomorphisms. The number of distinct isomorphic networks of six nodes is 156 , a significant reduction from the total number of networks, rendering the calculation of entropy computationally tractable.

In both plots, small magnitudes of the parameters give distributions with high entropy, as predicted.

\subsection{Nondegeneracy under general product transitions}

We can generalize the preceding discussion beyond the simple example model, by considering general transition distributions that factor over entries of $A_{i j}^{t}$ as follows. Suppose $\left\{\Psi_{k}\left(A^{t}, A^{t-1}\right)\right\}_{k}$ is a sequence of functions such that $\Psi_{k}\left(A^{t}, A^{t-1}\right)$ $=\sum_{i j} \Psi_{i j k}\left(A_{i j}^{t}, A^{t-1}\right)$ (i.e., satisfying (2)), where each $\Psi_{i j k}$ has range contained in $[-\beta, \beta]$ for some $\beta>0$, so that the range of $\Psi_{k}$ is in $[-n(n-1) \beta, n(n-1) \beta]$. Then we consider transition models of the form (1), with these $\Psi$ values. That is,

$$
\mathcal{P}\left(A^{t} \mid A^{t-1}, \boldsymbol{\theta}\right)=\frac{1}{Z\left(\boldsymbol{\theta}, A^{t-1}\right)} \exp \left\{\sum_{k} \theta_{k} \Psi_{k}\left(A^{t}, A^{t-1}\right)\right\} .
$$

Note that these models factor over the entries $A_{i j}^{t}$ given $A^{t-1}$.

Theorem 4.1. Let

$$
p=\frac{1}{\exp \left\{2 \beta \sum_{k}\left|\theta_{k}\right|\right\}+1} .
$$

For models of the form (4), the expected number of nonzero entries in $A^{t}$ is in the range

$$
[n(n-1) p, n(n-1)(1-p)],
$$

and the entropy can be lower bounded as

$$
H\left(A^{t}\right) \geq n(n-1)\left(p \log \frac{1}{p}+(1-p) \log \frac{1}{1-p}\right) .
$$

In particular, as long as $\sum_{k}\left|\theta_{k}\right|$ is not too large, this bound implies the entropy will be reasonably large. 
Proof. Since each $\Psi_{i j k}\left(A_{i j}^{t}, A^{t-1}\right) \in[-\beta, \beta]$, we can upper bound the probability of a particular entry $A_{i j}^{t}$ taking value 1 , given $A^{t-1}$, by

$$
\begin{aligned}
\mathcal{P}\left(A_{i j}^{t}=1 \mid A^{t-1}\right) & =\frac{\exp \left\{\sum_{k} \theta_{k} \Psi_{i j k}\left(1, A^{t-1}\right)\right\}}{\exp \left\{\sum_{k} \theta_{k} \Psi_{i j k}\left(1, A^{t-1}\right)\right\}+\exp \left\{\sum_{k} \theta_{k} \Psi_{i j k}\left(0, A^{t-1}\right)\right\}} \\
& \leq \frac{\exp \left\{\sum_{k}\left|\theta_{k}\right| \beta\right\}}{\exp \left\{\sum_{k}\left|\theta_{k}\right| \beta\right\}+\exp \left\{-\sum_{k}\left|\theta_{k}\right| \beta\right\}}=1-p
\end{aligned}
$$

and lower bound it by

$$
\begin{aligned}
\mathcal{P}\left(A_{i j}^{t}=1 \mid A^{t-1}\right) & =\frac{\exp \left\{\sum_{k} \theta_{k} \Psi_{i j k}\left(1, A^{t-1}\right)\right\}}{\exp \left\{\sum_{k} \theta_{k} \Psi_{i j k}\left(1, A^{t-1}\right)\right\}+\exp \left\{\sum_{k} \theta_{k} \Psi_{i j k}\left(0, A^{t-1}\right)\right\}} \\
& \geq \frac{\exp \left\{-\sum_{k}\left|\theta_{k}\right| \beta\right\}}{\exp \left\{\sum_{k}\left|\theta_{k}\right| \beta\right\}+\exp \left\{-\sum_{k}\left|\theta_{k}\right| \beta\right\}}=p .
\end{aligned}
$$

Since the conditional distribution given $A^{t-1}$ factors over the edges of $A^{t}$, the expected number of edges given $A^{t-1}$ is in this range, multiplied by $n(n-1)$. Since these bounds are independent of $A^{t-1}$, they also hold for the expectation under the marginal distribution of $A^{t}$. Similarly, as before we can lower bound the entropy under the marginal distribution of $A^{t}$ as

$$
\begin{aligned}
H\left(A^{t}\right) & \geq H\left(A^{t} \mid A^{t-1}\right) \geq \min _{A} H\left(A^{t} \mid A^{t-1}=A\right) \\
& =\min _{A} \sum_{i j} H\left(A_{i j}^{t} \mid A^{t-1}=A\right) \geq \sum_{i j} \min _{A} H\left(A_{i j}^{t} \mid A^{t-1}=A\right),
\end{aligned}
$$

and due to the bounds (5) and (6) on the conditional $\mathcal{P}\left(A_{i j}^{t}=1 \mid A^{t-1}\right)$, we have

$$
\min _{A} H\left(A_{i j}^{t} \mid A^{t-1}=A\right) \geq p \log \frac{1}{p}+(1-p) \log \frac{1}{1-p},
$$

so that

$H\left(A^{t}\right) \geq \sum_{i j} p \log \frac{1}{p}+(1-p) \log \frac{1}{1-p}=n(n-1)\left(p \log \frac{1}{p}+(1-p) \log \frac{1}{1-p}\right)$,

as claimed.

\section{Hypothesis testing: A case study}

As an example of how models of this type might be used in practice, we present a simple hypothesis testing application. Here we see the generality of this framework pay off, as we can use models of this type to represent a broad range of scientific hypotheses. The general approach to hypothesis testing in this framework is first to write down potential functions representing transitions one expects to be of some significance in a given population, next to write down potential 
functions representing the usual "background" processes (to serve as a null hypothesis), and third to plug these potentials into the model, calculate a test statistic, and compute a p-value.

The data involved in this example come from the United States $108^{\text {th }}$ Senate, having $n=100$ actors. Every time a proposal is made in the Senate, be it a bill, amendment, resolution, etc., a single Senator serves as the proposal's sponsor and there may possibly be several cosponsors. Given records of all proposals voted on in the full Senate, we create a sliding window of 100 consecutive proposals. For a particular placement of the window, we define a binary directed relation existing between two Senators if and only if one of them is a sponsor and the other a cosponsor for the same proposal within that window (where the direction is toward the sponsor). The data is then taken as evenly spaced snapshots of this sliding window, $A^{1}$ being the adjacency matrix for the first 100 proposals, $A^{2}$ for proposal 31 through 130 , and so on shifting the window by 30 proposals each time. In total, there are 14 observed networks in this series, corresponding to the first 490 proposals addressed in the $108^{\text {th }}$ Senate.

In this study, we propose to test the hypothesis that intraparty reciprocity is inherently stronger than interparty reciprocity. To formalize this, we use a model similar to the example given previously. The main difference is the addition of party membership indicator variables. Let $P_{i j}=1$ if the $i^{\text {th }}$ and $j^{\text {th }}$ actors are in the same political party, and 0 otherwise, and let $\bar{P}_{i j}=1-P_{i j}$. Define the following potential functions, representing stability, intraparty density, interparty density, ${ }^{1}$ overall reciprocity, intraparty reciprocity, and interparty reciprocity.

$$
\begin{aligned}
\Psi_{S}\left(A^{t}, A^{t-1}\right) & =\frac{1}{(n-1)} \sum_{i j}\left[A_{i j}^{t} A_{i j}^{t-1}+\left(1-A_{i j}^{t}\right)\left(1-A_{i j}^{t-1}\right)\right] \\
\Psi_{W D}\left(A^{t}, A^{t-1}\right) & =\frac{1}{(n-1)} \sum_{i j} A_{i j}^{t} P_{i j} \\
\Psi_{B D}\left(A^{t}, A^{t-1}\right) & =\frac{1}{(n-1)} \sum_{i j} A_{i j}^{t} \bar{P}_{i j} \\
\Psi_{R}\left(A^{t}, A^{t-1}\right) & =n\left[\sum_{i j} A_{j i}^{t} A_{i j}^{t-1}\right] /\left[\sum_{i j} A_{i j}^{t-1}\right] \\
\Psi_{W R}\left(A^{t}, A^{t-1}\right) & =n\left[\sum_{i j} A_{j i}^{t} A_{i j}^{t-1} P_{i j}\right] /\left[\sum_{i j} A_{i j}^{t-1} P_{i j}\right] \\
\Psi_{B R}\left(A^{t}, A^{t-1}\right) & =n\left[\sum_{i j} A_{j i}^{t} A_{i j}^{t-1} \bar{P}_{i j}\right] /\left[\sum_{i j} A_{i j}^{t-1} \bar{P}_{i j}\right]
\end{aligned}
$$

The null hypothesis supposes that the reciprocity observed in this data is the result of an overall tendency toward reciprocity amongst the Senators, regardless

\footnotetext{
${ }^{1}$ We split density to intra- and inter-party terms so as to factor out the effects on reciprocity of having higher intraparty density.
} 
of party. The alternative hypothesis supposes that there is a stronger tendency toward reciprocity among Senators within the same party than among Senators from different parties. Formally, the transition probability for the null hypothesis can be written as

$$
\mathcal{P}_{0}\left(A^{t} \mid A^{t-1}, \boldsymbol{\theta}^{(0)}\right)=\frac{1}{Z_{0}\left(\boldsymbol{\theta}^{(0)}, A^{t-1}\right)} \exp \left\{\sum_{j \in\{S, W D, B D, R\}} \theta_{j}^{(0)} \Psi_{j}\left(A^{t}, A^{t-1}\right)\right\},
$$

while the transition probability for the alternative hypothesis can be expressed as

$$
\mathcal{P}_{1}\left(A^{t} \mid A^{t-1}, \boldsymbol{\theta}^{(1)}\right)=\frac{1}{Z_{1}\left(\boldsymbol{\theta}^{(1)}, A^{t-1}\right)} \exp \left\{\sum_{j \in\{S, W D, B D, W R, B R\}} \theta_{j}^{(1)} \Psi_{j}\left(A^{t}, A^{t-1}\right)\right\} .
$$

For our test statistic, we use the likelihood ratio. To compute this, we compute the maximum likelihood estimators for each of these models, and take the ratio of the likelihoods. For the null hypothesis, the MLE is

$$
\left(\hat{\theta}_{S}^{(0)}=336.2, \hat{\theta}_{W D}^{(0)}=-58.0, \hat{\theta}_{B D}^{(0)}=-95.0, \hat{\theta}_{R}^{(0)}=4.7\right)
$$

with likelihood value of $e^{-9094.46}$. For the alternative hypothesis, the MLE is

$$
\left(\hat{\theta}_{S}^{(1)}=336.0, \hat{\theta}_{W D}^{(1)}=-58.8, \hat{\theta}_{B D}^{(1)}=-94.3, \hat{\theta}_{W R}^{(1)}=4.2, \hat{\theta}_{B R}^{(1)}=0.03\right)
$$

with likelihood value of $e^{-9088.96}$. The likelihood ratio statistic (null likelihood over alternative likelihood) is therefore about 0.0041. Because the null hypothesis is composite, determining the p-value of this result is more difficult, since we must determine the probability of observing a likelihood ratio at least this extreme under the null hypothesis for the parameter values $\boldsymbol{\theta}^{(0)}$ that maximize this probability. That is,

$$
\text { p-value }=\sup _{\boldsymbol{\theta}^{(0)}} \mathcal{P}_{0}\left\{\frac{\sup _{\hat{\boldsymbol{\theta}}^{(0)}} \mathcal{P}_{0}\left(A^{1}, \ldots, A^{14} \mid \hat{\boldsymbol{\theta}}^{(0)}\right)}{\sup _{\hat{\boldsymbol{\theta}}^{(1)}} \mathcal{P}_{1}\left(A^{1}, \ldots, A^{14} \mid \hat{\boldsymbol{\theta}}^{(1)}\right)} \leq 0.0041 \mid \boldsymbol{\theta}^{(0)}\right\} .
$$

In general this seems not to be tractable to analytic solution, so we employ a genetic algorithm to perform the unconstrained optimization, and approximate the probability for each parameter vector by sampling. That is, for each parameter vector $\boldsymbol{\theta}^{(0)}$ (for the null hypothesis) in the GA's population on each iteration, we sample a large set of sequences from the joint distribution. For each sequence, we compute the MLE under the null hypothesis and the MLE under the alternative hypothesis, and then calculate the likelihood ratio and compare it to the observed ratio. We calculate the empirical frequency with which the likelihood ratio is at most 0.0041 in the set of sampled sequences for each vector $\boldsymbol{\theta}^{(0)}$, and use this as the objective function value in the genetic algorithm. Mutations consist of adding Gaussian noise (with variance decreasing on each 
iteration), and recombination is performed as usual. Full details of the algorithm are omitted for brevity (see (Mitchell, 1996) for an introduction to GAs). The resulting approximate $\mathrm{p}$-value we obtain by this optimization procedure is 0.024 .

Because it has the form (2), this model has the property that we can compute the likelihoods and derivatives thereof analytically. In particular, in models of this form, we can compute likelihoods and perform Newton-Raphson optimization directly, without the need of sampling-based approximations. However, in general this might not be the case. For situations in which one cannot tractably compute the likelihoods, an alternative possibility is to use bounds on the likelihoods. Specifically, one can obtain an upper bound on the likelihood ratio statistic by dividing an upper bound on the null likelihood by a lower bound on the alternative likelihood. When computing the p-value, one can use a lower bound on the ratio by dividing a lower bound on the null likelihood by an upper bound on the alternative likelihood. See (Opper and Saad, 2001; Wainwright et al., 2005) for examples of how such bounds on the likelihood can be tractably attained, even for intractable models.

In practice, the problem of formulating an appropriate model to encode one's hypothesis is not well-posed. One general approach which seems intuitively appealing is to write down the types of motifs or patterns one expects to find in the data, and then specify various other patterns which one believes those motifs could likely transition to (or would likely not transition to) under the alternative hypothesis. For example, perhaps one believes that densely connected regions of the network will tend to become more dense and clique-like over time, so that one might want to write down a potential representing the transition of, say, k-cliques to more densely connected structures.

\section{Classification: A case study}

One can additionally consider using these temporal models for classification.

Specifically, consider a transductive learning problem in which each actor has a static class label, but the learning algorithm is only allowed to observe the labels of some random subset of the population. The question is then how to use the known label information, in conjunction with observations of the network evolving over time, to accurately infer the labels of the remaining actors whose labels are unknown.

As an example of this type of application, consider the alternative hypothesis model from the previous section (model 1), in which each Senator has a class label (party affiliation). We can slightly modify the model so that the party labels are no longer constant, but random variables drawn independently from a known multinomial distribution. Assume we know the party affiliations of a randomly chosen 50 Senators. This leaves 50 Senators with unknown affiliations. If we knew the parameters $\boldsymbol{\theta}$, we could predict these 50 labels by sampling from the posterior distribution and taking the mode for each label. However, since both the parameters and the 50 labels are unknown, this is not possible. Instead, we can perform Expectation Maximization to jointly infer the maximum likelihood estimator $\hat{\boldsymbol{\theta}}$ for $\boldsymbol{\theta}$ and the posterior mode given $\hat{\boldsymbol{\theta}}$. 
Specifically, let us assume the two class labels are Democrat and Republican, and we model these labels as independent Bernoulli(0.5) random variables. The distribution on the network sequence given that all 100 labels are fully observed is the same as given in the previous section. Since one can compute likelihoods in this model, sampling from the posterior distribution of labels given the network sequence is straightforward using Gibbs sampling. We can therefore employ a combination of MCEM and Generalized EM algorithms (call it MCGEM) (McLachlan and Krishnan, 1997) with this model to infer the party labels as follows. In each iteration of the algorithm, we sample from the posterior distribution of the unknown class labels under the current parameter estimates given the observed networks and known labels, approximate the expectation of the gradient and Hessian of the log likelihood using the samples, and then perform a single Newton-Raphson update using these approximations.

We run this algorithm on the $108^{\text {th }}$ Senate data from the previous section. We randomly select 50 Senators whose labels are observable, and take the remaining Senators as having unknown labels. As mentioned above, we assume all Senators are either Democrat or Republican; Senator Jeffords, the only independent Senator, is considered a Democrat in this model. We run the MCGEM algorithm described above to infer the maximum likelihood estimator $\hat{\boldsymbol{\theta}}$ for $\boldsymbol{\theta}$, and then sample from the posterior distribution over the 50 unknown labels under that maximum likelihood distribution, and take the sample mode for each label to make a prediction.

The predictions of this algorithm are correct on $70 \%$ of the 50 Senators with unknown labels. Additionally, it is interesting to note that the parameter values the algorithm outputs $\left(\hat{\theta}_{S}=336.0, \hat{\theta}_{W D}=-59.7, \hat{\theta}_{B D}=-96.0, \hat{\theta}_{W R}=\right.$ $\left.3.8, \hat{\theta}_{B R}=0.28\right)$ are very close (Euclidean distance 2.0$)$ to the maximum likelihood estimator obtained in the previous section (where all class labels were known). Compare the above accuracy score with a baseline predictor that always predicts Democrat, which would get $52 \%$ correct for this train/test split, indicating that this statistical model of network evolution provides at least a somewhat reasonable learning bias. However, there is clearly room for improvement in the model specification, and it is not clear whether modeling the evolution of the graph is actually of any benefit for this particular example. For example, after collapsing this sequence of networks into a single weighted graph with edge weights equal to the sum of edge weights over all graphs in the sequence, running Thorsten Joachims' Spectral Graph Transducer algorithm (Joachims, 2003) gives a $90 \%$ prediction accuracy on the Senators with unknown labels. These results are summarized in Table 1 . Further investigation is needed into what

TABle 1

Summary of classification results

\begin{tabular}{|l|r|}
\hline Method & Accuracy \\
\hline Baseline & $52 \%$ \\
\hline Temporal Model & $70 \%$ \\
\hline SGT & $90 \%$ \\
\hline
\end{tabular}


types of problems can benefit from explicitly modeling the network evolution, and what types of models are most appropriate for basing a learning bias on.

\section{Assessing statistic importance and quality of fit: A case study}

In this section, we use TERGMs to model the network transitions of the $108^{\text {th }}$ U.S. Senate network, described in Section 5. The dynamic network has 100 nodes and 12 time points ${ }^{2}$. We perform two types of experiments here: the first is simply to assess which statistics are important for modeling the network transitions, by observing the magnitudes of the estimated parameters, and the second assesses the quality of fit of a model with a cross-validation experiment.

We start with including three statistics: Density, Stability, and Reciprocity. The estimated parameters are plotted in Figure 3. We have estimated 11 sets of model parameters, so each box plot in a subplot contains 11 values. Judging by the magnitudes of the weights, we can see that Density and Stability play big roles, whereas Reciprocity plays a minor role in this case.

The 3-statistic TERGM model is so simple that we would not expect it to have enough modeling power. Therefore, we introduce some 3 -node statistics ${ }^{3}$ and expand to include 7 statistics in the model. The 4 new statistics are Transitivity, Reverse-Transitivity, Co-Supported, and Co-Supporting, which are illustrated in Figure 4. Transitivity has been explained in earlier sections. Reverse-Transitivity means that if person $\mathrm{B}$ supports person $\mathrm{C}$ and person $\mathrm{C}$ supports person $\mathrm{A}$ at time $(t-1)$, then it is likely that person A will support person B at time $t$. CoSupported says that if both A and B are supported by a third person at time $t$, then it is likely that person A will support person B at time $t$. Co-Supporting is defined similarly. In all of the cases, we are looking at the influence from the previous time point on the link from A to B at time $t$.

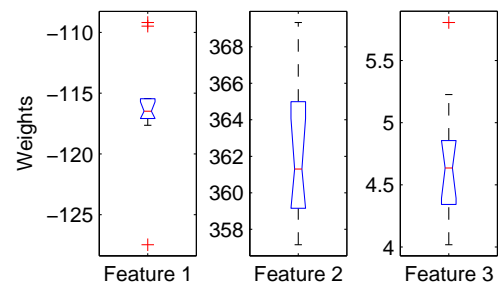

FIG 3. Estimated parameter values (weights) for a TERGM with 3 statistics (features). Feature 1 is Density; Feature 2 is Stability; Feature 3 is Reciprocity.

\footnotetext{
${ }^{2}$ We have removed the first two time points from the original 14-step series of Section 5 , due to outlier behavior in the initial two time points. This behavior is explained by an initial surge in activity when the Senate reconvenes after a vacation, but is not part of the usual "stationary" behavior, so we chose to exclude it when evaluating the quality of fit.

${ }^{3}$ We call Density, Stability, and Reciprocity 2-node features because their decomposed forms only involve two nodes.
} 


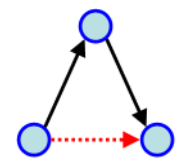

Transitivity
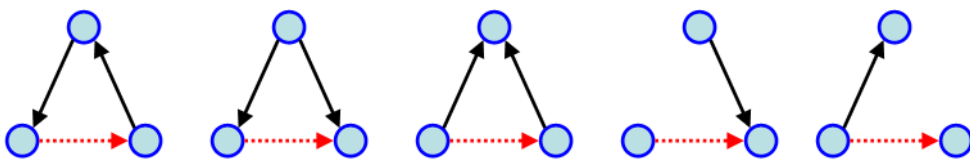

Reverse- Co-Supported Co-Supporting Popularity
Transitivity

FIG 4. Graph illustrations of six 3-node statistics corresponding to Features 4-9 in Figure 5 and 6. Blue circles are nodes; black solid arrows represent links (or a supporting relationship) at time $(t-1)$; red dotted arrows represent an edge at time $t$.
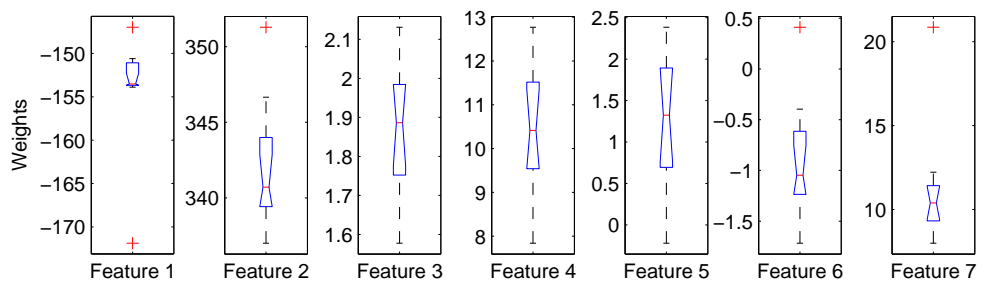

FIG 5. Estimated parameter values (weights) for a TERGM with 7 statistics (features).

More formally, the new statistics are defined as

$$
\begin{aligned}
\Psi_{R T}\left(A^{t}, A^{t-1}\right) & =n\left[\sum_{i j k} A_{j k}^{t-1} A_{k i}^{t-1} A_{i j}^{t}\right] /\left[\sum_{i j k} A_{j k}^{t-1} A_{k i}^{t-1}\right] \\
\Psi_{C S d}\left(A^{t}, A^{t-1}\right) & =n\left[\sum_{i j k} A_{k i}^{t-1} A_{k j}^{t-1} A_{i j}^{t}\right] /\left[\sum_{i j k} A_{k i}^{t-1} A_{k j}^{t-1}\right] \\
\Psi_{C S g}\left(A^{t}, A^{t-1}\right) & =n\left[\sum_{i j k} A_{i k}^{t-1} A_{j k}^{t-1} A_{i j}^{t}\right] /\left[\sum_{i j k} A_{i k}^{t-1} A_{j k}^{t-1}\right] .
\end{aligned}
$$

Figure 5 shows the parameter values for the TERGM model with these 7 statistics. Among the 4 new statistics, Transitivity and Co-Supporting are major contributors, while Reverse-Transitivity and Co-Supported are neglectable. The effectiveness of Transitivity is intuitive, but the big contrast between the weights for Co-Supporting and for Co-Supported could be intricate. It can be explained by the nature of the data. Each proposal has a single sponsor and possibly multiple co-sponsors. Therefore, each Senator is likely to be a sponsor (supported by others) for few proposals, while she or he could potentially be a co-sponsor (supporter) for many more proposals. When the Co-Supporting case happens, it is likely that the two Senators supported a third Senator on the same proposal, which suggests a shared position on the issue, which could further lead to a cooperation on another proposal at a later time. In contrast, when the Co-Supported situation happens, it is certain that the two Senators are supported by a third senator on different proposals. ${ }^{4}$ Although they are

\footnotetext{
${ }^{4}$ Links corresponding to a proposal are pointing to a single node, since there is only one sponsor for each proposal.
} 

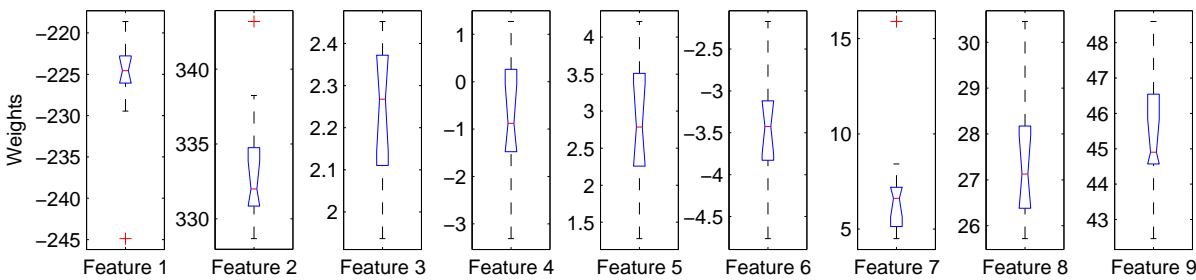

FIG 6. Estimated parameter values (weights) for a TERGM with 9 statistics (features).

co-sponsored by a same Senator, these proposals can be in very different areas, which does not necessarily suggest a common interest for the two sponsors.

Next, we add two more 3-node statistics, Popularity and Generosity, to the model to have a set of 9 statistics. As illustrated in Figure 4, Popularity says that if one has a supporter, she or he is likely to have another supporter. Generosity can be understood in the same manner. More formally, they are defined as

$$
\begin{aligned}
& \Psi_{P}\left(A^{t}, A^{t-1}\right)=n\left[\sum_{i j k} A_{k j}^{t-1} A_{i j}^{t}\right] /\left[\sum_{i j k} A_{k j}^{t-1}\right] \\
& \Psi_{G}\left(A^{t}, A^{t-1}\right)=n\left[\sum_{i j k} A_{i k}^{t-1} A_{i j}^{t}\right] /\left[\sum_{i j k} A_{i k}^{t-1}\right] .
\end{aligned}
$$

Figure 6 shows the estimated parameter values for the TERGM with 9 statistics. Both Popularity and Generosity have significant weights, while Transitivity has a weight with low magnitude. In some sense, the Transitivity statistic can be viewed as Popularity plus Generosity with some constraints. This plot indicates that the importance of Popularity and Generosity was the indirect cause of our emphasis on Transitivity in the simpler models, since in a model containing these statistics, Transitivity becomes irrelevant.

Next, we heuristically evaluate the quality of fit of the model using a crossvalidation style experiment. Hunter, Goodreau, and Handcock (2008) have recently proposed a method for evaluating goodness-of-fit for ERGMs, modeling a single network. The idea is to hold out one statistic and fit the parameters for the remaining statistics, and then determine how well the distribution of the held-out statistic under the fit parameters captures its observed value from the data. Although a similar approach should be possible in our present setting, we have chosen to take a slightly different approach to assessing fitness. Instead, we propose holding out one of the temporal transitions. Specifically, for each of the time points $t$ (except $t=1$ ), we estimate the entire set of parameters for a TERGM to fit all the observed transitions except the transition from time point $t-1$ to time point $t$. We then assess how well the fit parameters predict the transition of the statistics' values for the held-out transition, as follows. We sample a number of networks from the conditional distribution over the network at time $t$ given the observed network at time $t-1$, under the estimated parameters. Then we compare the $\Psi\left(A^{t}, A^{t-1}\right)$ statistic values from the sampled $A^{t}$ networks to the $\Psi\left(A^{t}, A^{t-1}\right)$ statistic values from the true $A^{t}$ network. We 


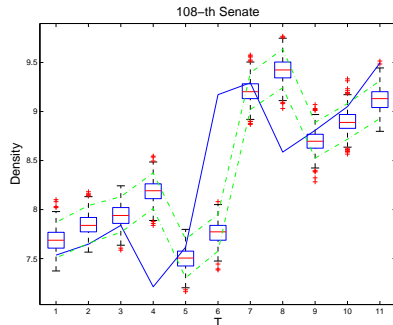

108-th Senate

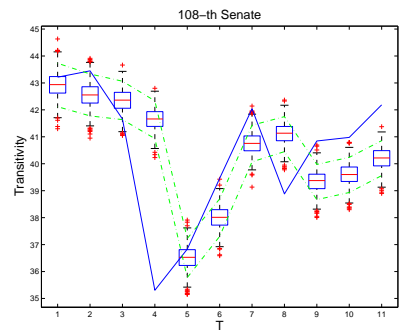

108-th Senate
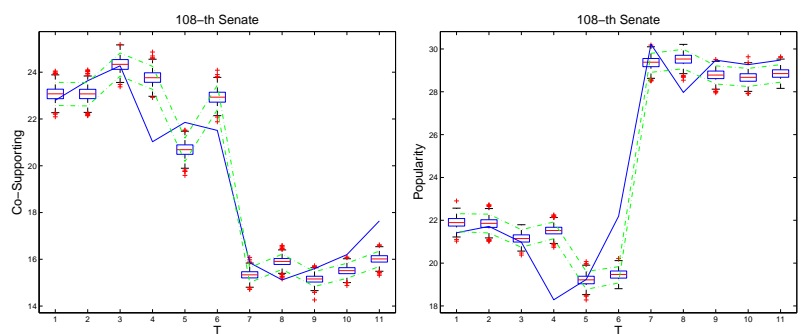

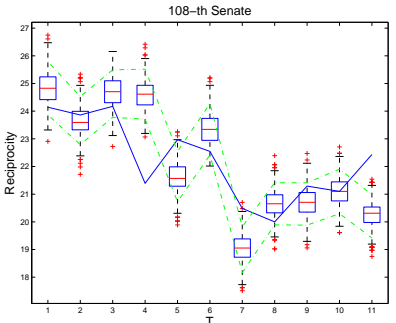

108-th Senate

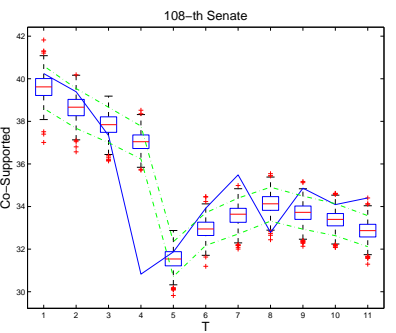

108-th Senate

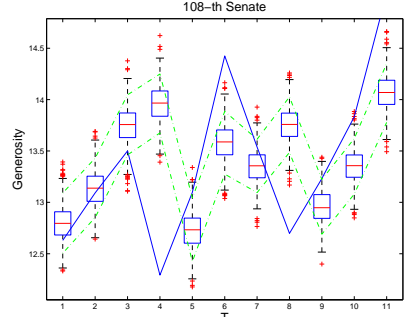

FIG 7. Statistic values of real networks and sampled networks based on a TERGM with 9 statistics. The comparisons are grouped by statistic. Blue solid lines indicate the observed (true) network statistics. Box plots are for the sampled networks (in the described crossvalidation experiments) and green dotted lines indicate 5- and 95-percentiles.

repeat this entire process for each $t>1$ to generate our plots. The results of this comparison reflect relatively how well this TERGM models the transitions, given that we are committed to a Markov assumption for the transitions.

Figure 7 presents the comparison of statistic values between ground-truth and sampled networks from the estimated TERGMs from the cross-validation process described above. For a few statistics, the blue lines lie within the green lines (i.e., in the range of sampled networks) for most time points, which means that the model does a fairly good job of predicting the change in statistic values: for example, Reciprocity, Reverse-Transitivity, Co-Supported, Co-Supporting, and Popularity. It is worth noting that we can even capture some sharp changes with these models: for instance, Reverse-Transitivity, Co-Supporting, and Popularity at time point 7 (the last of these is particularly dramatic). This is somewhat surprising, as it intuitively seems like sharp changes might be quite difficult to predict with a Markov assumption and such simple statistics. On the other hand, not all statistics are predicted well, such as Stability, where the predictions are quite poor; as such, there is clearly room for improvement in the design of statistics to more accurately model time-evolving networks. 


\section{Future work}

If we think of this type of model as describing a process giving rise to the networks one observes in reality, then one can think of a single network observation as a snapshot of this Markov chain at that time point. Traditionally one would model a network at a single time point using an ERGM. However, in light of the degeneracy issues found in ERGMs, and the lack thereof for the temporal models with product conditional distributions, it seems worthwhile to investigate modeling a single network as the end-point of an unobservable sequence. Directly modeling this with latent variables would seem to make inference computationally difficult. However, it may be possible to indirectly model this by studying the stationary distribution of these Markov chains. To our knowledge, it remains an open problem to directly specify the family of stationary distributions that a given TERGM corresponds to.

Moving forward, we hope to move beyond these ERG-inspired models toward models that incorporate latent variables, which may also evolve over time with the network. For example, it may often be the case that the phenomena represented in data can most easily be described by imagining the existence of unobserved groups or factions, which form, dissolve, merge and split as time progresses. The flexibility of the ERG models and the above temporal extensions allows a social scientist to "plug in" his or her knowledge into the formulation of the model, while still providing general-purpose estimation algorithms to find the right trade-offs between competing and complementary factors in the model. We would like to retain this flexibility in formulating a general family of models that include evolving latent variables in the representation, so that the researcher can "plug in" his or her hypotheses about latent group dynamics, evolution of unobservable actor attributes, or a range of other possible phenomena into the model representation. At the same time, we would like to preserve the ability to provide a "black box" inference algorithm to determine the parameter and variable values of interest to the researcher, as can be done with ERGMs and now TERGMs.

\section{Acknowledgments}

We thank Stephen Fienberg and all participants in the statistical network modeling discussion group at Carnegie Mellon for helpful comments and feedback. This work is support in part by funding from NSF IIS-0713379, NSF DBI0546594 Career Award, ONR N000140910758, DARPA NBCH1080007, and an Alfred P. Sloan Fellowship awarded to EPX.

\section{References}

Airoldi, E. M., Blei, D. M., Fienberg, S. E., and Xing, E. P. (2008). Mixed membership stochastic blockmodels. Journal of Machine Learning Research, 9, 1981-2014. 
Carreira-Perpignán, M. and Hinton, G. E. (2005). On contrastive divergence learning. In Artificial Intelligence and Statistics.

Frank, O. and Strauss, D. (1986). Markov graphs. Journal of the American Statistical Association, 81. MR0860518

Geyer, C. and Thompson, E. (1992). Constrained Monte Carlo maximum likelihood for dependent data. Journal of the Royal Statistical Society, B, 54(3), 657-699. MR1185217

Handcock, M. S. (2003). Assessing Degeneracy in Statistical Models of Social Networks. Center for Statistics and the Social Sciences, University of Washington, Working Paper No. 39.

Handcock, M. S., Raftery, A. E., and Tantrum, J. (2007). Model-based clustering for social networks. Journal of the Royal Statistical Society A, 170, 301-354. MR2364300

Hunter, D. R., Goodreau, S. M., and Handcock, M. S. (2008). Goodness of fit of social network models. Journal of the American Statistical Association, 103(1), 248-258. MR2394635

JoACHims, T. (2003). Transductive learning via spectral graph partitioning. In Proceedings of the International Conference on Machine Learning.

McLachlan, G. and Krishnan, T. (1997). The EM algorithm and Extensions. Wiley. MR1417721

Mitchell, M. (1996). An Introduction to Genetic Algorithms. MIT Press.

Opper, M. and SAAD, D., editors (2001). Advanced Mean Field Methods: Theory and Practice. MIT Press. MR1863214

Robins, G. L. and Pattison, P. E. (2001). Random graph models for temporal processes in social networks. Journal of Mathematical Sociology, 25, $5-41$.

Robins, G. L. and Pattison, P. E. (2005). Interdependencies and social processes: Generalized dependence structures. In P. Carrington, J. Scott, and S. Wasserman, editors, Models and Methods in Social Network Analysis. Cambridge University Press.

SniJders, T. (2006). Statistical methods for network dynamics. In S. L. et al., editor, Proceedings of the XLIII Scientific Meeting, Italian Statistical Society, pages 281-296.

Snijders, T. A. B. (2002). Markov Chain Monte Carlo estimation of exponential random graph models. Journal of Social Structure, $\mathbf{3}(2)$.

Wainwright, M., JaAkkola, T., and Willsky, A. (2005). A new class of upper bounds on the log partition function. IEEE Trans. on Information Theory, $\mathbf{5 1}(7)$.

Wasserman, S. and Robins, G. (2005). An introduction to random graphs, dependence graphs, and p*. In P. Carrington, J. Scott, and S. Wasserman, editors, Models and Methods in Social Network Analysis. Cambridge University Press. 